\title{
Minimum Segment Drawings of Series-Parallel Graphs with the Maximum Degree Three
}

(Extended Abstract)

\author{
Md. Abul Hassan Samee ${ }^{1}$, Md. Jawaherul Alam², \\ Muhammad Abdullah Adnan ${ }^{2}$, and Md. Saidur Rahman ${ }^{2}$ \\ ${ }^{1}$ Department of Computer Science, University of Illinois at Urbana-Champaign, IL, USA \\ ${ }^{2}$ Department of Computer Science and Engineering, Bangladesh University of Engineering and \\ Technology, Dhaka, Bangladesh \\ samee1@uiuc.edu, jawaherul@yahoo.com, adnan@cse.buet.ac.bo, \\ saidurrahman@cse. buet.ac.bd
}

\begin{abstract}
A minimum segment drawing $\Gamma$ of a planar graph $G$ is a straight line drawing of $G$ that has the minimum number of segments among all straight line drawings of $G$. In this paper, we give a linear-time algorithm for computing a minimum segment drawing of a series-parallel graph with the maximum degree three. To the best of our knowledge, this is the first algorithm for computing minimum segment drawings of an important subclass of planar graphs.
\end{abstract}

\section{Introduction}

A straight line drawing $\Gamma$ of a planar graph $G$ is a planar drawing where each vertex $u$ of $G$ is mapped to a point $p(u)$ in the plane and each edge $e=(u, v)$ of $G$ is drawn as a line segment $l(e)$ closed between the points $p(u)$ and $p(v)$. A line segment $L$ in a straight line drawing $\Gamma$ is said to be a maximal line segment in $\Gamma$ if $L$ is formed by a maximal set of line segments $l_{1}, l_{2}, \ldots, l_{k}$ such that each pair $l_{i}$ and $l_{i+1}$ has a common end point in $\Gamma(0<i<k)$. In the remainder of this paper, we simply use the term segment to refer a maximal line segment in a straight line drawing. A straight line drawing $\Gamma$ of $G$ is called a minimum segment drawing of $G$ if $\Gamma$ has the minimum number of segments among all possible straight line drawings of $G$. For example, the graph $G$ in Fig.1 (a) can be drawn with seven segments as shown in Fig.11(b). Another drawing of $G$ with five segments is shown in Fig.1(c). One can easily verify that unless we change the embedding of $G$ in Fig. 1 a), it is impossible to draw $G$ with less than five segments. However, if we consider the embedding shown in Fig. 1(d), then $G$ can be drawn with four segments as shown in Fig. 1 e). One can also verify that, it is not possible to draw any embedding of $G$ with less than four segments. Thus, the drawing of $G$ in Fig. 1(e) is a minimum segment drawing of $G$.

The problem of computing straight line drawings of planar graphs has been studied for long with various application specific objectives [2|3|5|89 10]. Recently, Dujmović et al. have studied this problem with a new objective of minimizing the number of 


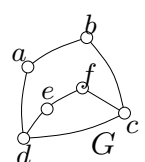

(a)

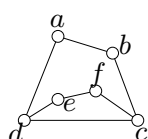

(b)

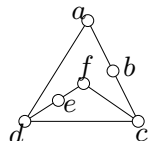

(c)

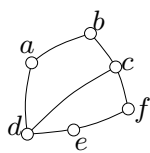

(d)

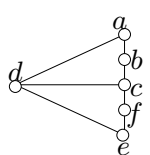

(e)

Fig. 1. (a) The graph $G$, (b) a drawing of $G$ on seven segments, (c) a drawing of $G$ on five segments, (d) another embedding of $G$, and (e) a minimum segment drawing of $G$

segments in a drawing [1], and the insightful results presented in their work have established a new line of research henceforth. However, as their results suggest, this problem is quite difficult for most of the non-trivial graph classes. For most of these cases, bounds have been given on the number of segments in a drawing, but no algorithm is known so far for computing a minimum segment drawing. For example, although Dujmović et al. have provided an algorithm for computing minimum segment drawings of trees, no algorithm is known for biconnected and triconnected planar graphs. The problem has also been studied for plane graphs. Although dealing with plane graphs is typically easier than dealing with planar graphs, no algorithm is known for computing minimum segment drawings of biconnected and triconnected plane graphs as well. Even for degree restricted cases of plane graphs, e.g., for plane triconnected cubic graphs, no algorithm has yet been devised for computing minimum segment drawings.

In this paper, we study the minimum segment drawing problem for series-parallel graphs with the maximum degree three. For such a graph $G$, we give linear-time algorithms for choosing such an embedding of $G$ that admits a straight-line drawing on the minimum number of segments, and for computing a minimum segment drawing of $G$. The rest of this paper is organized as follows. In Section 2 we give some definitions and present our primary results. In Section 3 we give a linear-time algorithm for computing a minimum segment drawing of a biconnected series-parallel graph with the maximum degree three. In Section 4 we briefly illustrate how our idea from Section 3 can be extended to compute minimum segment drawing of a series-parallel graph which is not necessarily biconnected. Finally Section 5 is a conclusion.

\section{Preliminaries}

In this section we give some relevant definitions and present our preliminary results. For basic graph theoretic and graph drawing related definitions we refer to [4].

A graph $G=(V, E)$ is called a series-parallel graph (with source $s$ and sink $t$ ) if either $G$ consists of a pair of vertices $s$ and $t$ connected by a single edge, or there exist two series-parallel graphs $G_{i}=\left(V_{i}, E_{i}\right), i=1,2$, with source $s_{i}$ and sink $t_{i}$ such that $V=V_{1} \cup V_{2}, E=E_{1} \cup E_{2}$, and either $(i) s=s_{1}, t_{1}=s_{2}$ and $t=t_{2}$, or (ii) $s=s_{1}=s_{2}$ and $t=t_{1}=t_{2}$ [6]. A pair $\{u, v\}$ of vertices of a connected graph $G$ is a split pair if there exist two subgraphs $G_{1}=\left(V_{1}, E_{1}\right)$ and $G_{2}=\left(V_{2}, E_{2}\right)$ such that: (i) $V=V_{1} \cup V_{2}, V_{1} \cap V_{2}=\{u, v\}$; and (ii) $E=E_{1} \cup E_{2}, E_{1} \cap E_{2}=\emptyset$, $\left|E_{1}\right| \geq 1,\left|E_{2}\right| \geq 1$. Thus every pair of adjacent vertices of $G$ is a split pair of $G$. A split component of a split pair $\{u, v\}$ is either an edge $(u, v)$ or a maximal connected subgraph $H$ of $G$ such that $\{u, v\}$ is not a split pair of $H$. 


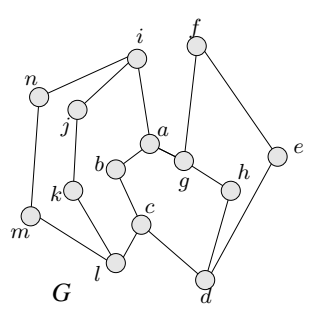

(a)

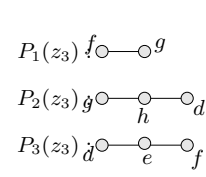

(d)

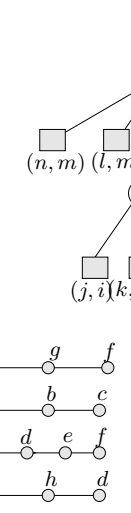

(e)

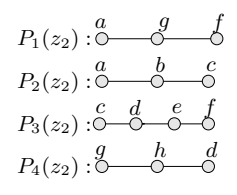

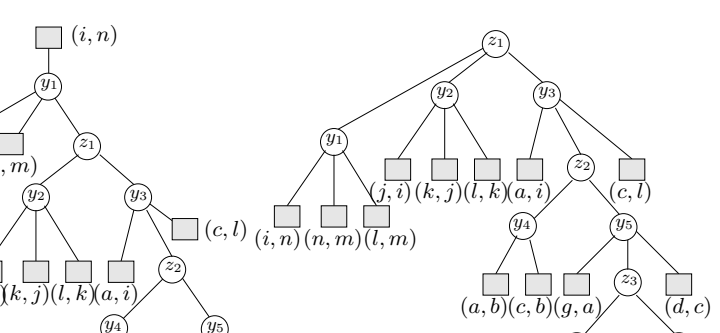

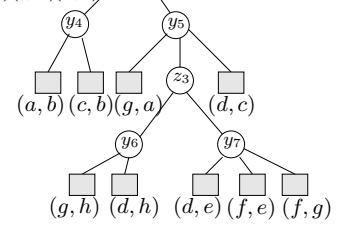

(b)

Fig. 2. (a) A biconnected series-parallel graph $G$ with $\Delta(G)=3$, (b) $S P Q$-tree $\mathcal{T}$ of $G$ with respect to reference edge $(i, n)$, (c) $S P Q$-tree $\mathcal{T}$ of $G$ with $P$-node $z_{1}$ as the root, (d) the three core paths of node $z_{3}$, and (e) the four core paths of node $z_{2}$

Let $G$ be a biconnected series-parallel graph. The $S P Q$-tree $\mathcal{T}$ of $G$ with respect to a reference edge $e=(s, t)$ is a recursive decomposition of $G$ induced by its split pairs [6]. $\mathcal{T}$ is a rooted ordered tree whose nodes are of three types: $S, P$ and $Q$. Each node $x$ of $\mathcal{T}$ corresponds to a subgraph of $G$, called its pertinent graph $G(x)$. Tree $\mathcal{T}$ is recursively defined as follows.

(i) Trivial Case: In this case, $G$ consists of two parallel edges $e$ and $e^{\prime}$ joining $s$ and $t$. The tree $\mathcal{T}$ consists of a single $Q$-node $x$. The pertinent graph $G(x)$ consists of only the edge $e^{\prime}$. (ii) Parallel Case: In this case, the split pair $\{s, t\}$ has three or more split components $G_{0}, G_{1}, \ldots, G_{k}(k \geq 2)$, and $G_{0}$ consists of only a reference edge $e=(s, t)$. The root of $\mathcal{T}$ is a $P$-node $x$. The pertinent graph $G(x)=G_{1} \cup G_{2} \cup \cdots \cup G_{k}$. (iii) Series Case: In this case, the split pair $\{s, t\}$ has exactly two split components, and one of them consists of the reference edge $e$. One may assume that the other split component has cut-vertices $c_{1}, c_{2}, \ldots c_{k-1}(k \geq 2)$, that partition the component into its blocks $G_{1}, G_{2}, \ldots, G_{k}$ in this order from $s$ to $t$. Then the root of $\mathcal{T}$ is an $S$-node $x$. The pertinent graph $G(x)$ of node $x$ is a union of $G_{1}, G_{2}, \ldots, G_{k}$. In Fig. 2 we have illustrated the concept of representing the recursive decomposition of a given biconnected series-parallel graph through an $S P Q$-tree. In each of the cases mentioned above, we call the edge $e$ the reference edge of node $x$. Except for the trivial case, node $x$ of $\mathcal{T}$ has children $x_{1}, x_{2}, \ldots, x_{k}$ in this order; $x_{i}$ is the root of the $S P Q$-tree of graph $G\left(x_{i}\right) \cup e_{i}$ with respect to the reference edge $e_{i}, 1 \leq i \leq k$. We call edge $e_{i}$ the reference edge of node $x_{i}$, and call the endpoints of edge $e_{i}$ the poles of node $x_{i}$. The tree obtained so far has a $Q$-node associated with each edge of $G$, except the reference edge $e$. We complete the $S P Q$-tree $\mathcal{T}$ by adding a $Q$-node, representing the reference edge $e$, and making it the parent of $x$ so that it becomes the root of $\mathcal{T}$. One can easily modify $\mathcal{T}$ to an $S P Q$-tree $\mathcal{T}^{\prime}$ with an arbitrary $P$-node as the root as illustrated in Fig. $2(\mathrm{e})$. In the remainder of this paper, we consider $S P Q$-trees having $P$-nodes as their roots. Based on the assumption that $\Delta(G)=3$, the following facts were mentioned in [6]. 
Fact 1. Let $(s, t)$ be the reference edge of an $S$-node $x$ of $\mathcal{T}$, and let $x_{1}, x_{2}, \ldots, x_{k}$ be the children of $x$ in this order from $s$ to $t$. Then the following $(i)-($ iii) hold. $(i)$ Each child $x_{i}$ of $x$ is either a P-node or a $Q$-node; (ii) both $x_{1}$ and $x_{k}$ are $Q$-nodes; and (iii) $x_{i-1}$ and $x_{i+1}$ must be $Q$-nodes if $x_{i}$ is a $P$-node where $2 \leq i \leq k-1$.

Fact 2. The root $P$-node of $\mathcal{T}$ has exactly three children and each non-root $P$-node of $\mathcal{T}$ has exactly two children. For a non-root $P$-node $x$ in $\mathcal{T}$, either both the children of $x$ are $S$-nodes, or one child of $x$ is an $S$-node and the other child of $x$ is a $Q$-node.

A node $x$ in $\mathcal{T}$ is primitive if $x$ does not have any descendant $P$-node in $\mathcal{T}$. We define the height of a primitive $P$-node to be zero. The height of any other $P$-node is $(i+1)$ if the maximum of the heights of its descendant $P$-nodes is $i$. For two given $P$-nodes $x$ and $z$ in $\mathcal{T}$, we say that $z$ is a child $P$-node of $x$ if there is an $S$-node $y$ in $\mathcal{T}$ such that $y$ is a child of $x$ and $z$ is a child of $y$ in $\mathcal{T}$.

Let $G$ be a biconnected series-parallel graph with $\Delta(G)=3$. Let $G^{\prime}$ be the plane graph corresponding to a straight line drawing $\Gamma$ of $G$. Let $\mathcal{T}^{\prime}$ be an $S P Q$-tree of $G^{\prime}$, and $r$ be such a $P$-node in $\mathcal{T}^{\prime}$ that the poles of $r$ appear on the outerface of $\Gamma$. An $S P Q$-tree of $G$ corresponding to $\Gamma$ is the $S P Q$-tree obtained by considering $\mathcal{T}^{\prime}$ rooted at $r$. We use $\mathcal{T}_{\Gamma}$ to denote an $S P Q$-tree of $G$ corresponding to a drawing $\Gamma$ of $G$. For a node $x$ in $\mathcal{T}_{\Gamma}$, let $P_{x}$ and $N_{x}$ denote the number of $P$-nodes and primitive $P$-nodes in the subtree of $\mathcal{T}_{\Gamma}$ rooted at $x$. If $x$ is a non-root $P$-node, then let $y$ and $y^{\prime}$ denote the two children of $x$ in $\mathcal{T}_{\Gamma}$. Let $p$ and $q$ denote the number of child $P$-and $Q$-nodes respectively of the node $y$ in $\mathcal{T}_{\Gamma}$. Similarly, let $p^{\prime}$ and $q^{\prime}$ denote the number of child $P$ - and $Q$-nodes respectively of $y^{\prime}$ in $\mathcal{T}_{\Gamma}$. Let $z_{i}$ denote the $i$-th child $P$-node of $y$ in $\mathcal{T}_{\Gamma}$ and $e_{i}$ denote the edge corresponding to the $i$-th child $Q$-node of $y$ in $\mathcal{T}_{\Gamma}$. Similarly, let $z_{i}^{\prime}$ denote the $i$-th child $P$-node of $y^{\prime}$ in $\mathcal{T}_{\Gamma}$ and $e_{i}^{\prime}$ denote the edge corresponding to the $i$-th child $Q$-node of $y^{\prime}$ in $\mathcal{T}_{\Gamma}$. For each non-root $P$-node $x$ of $\mathcal{T}_{\Gamma}$, we now define the core paths of $G(x)$ as follows. If $x$ is a primitive $P$-node, then let $q \geq q^{\prime}$. We then define three core paths $P_{i}(x)(1 \leq i \leq 3)$ of $G(x)$ as $P_{1}(x)=e_{1}, P_{2}(x)=G\left(y^{\prime}\right)$ and $P_{3}(x)=\bigcup_{i=2}^{q} e_{i}$, as shown in Fig. 2 d). Otherwise, $x$ is not primitive, and we consider the following two subcases. If either of the two nodes $y$ and $y^{\prime}$ has at least two child $P$-nodes then we assume that $y$ is such a node, otherwise we assume that $p \geq p^{\prime}$ and proceed as follows. Let $e_{j}$ and $e_{k}$ denote the edges corresponding to the $Q$-nodes immediately preceding $z_{1}$ and $z_{p}$, respectively in $\mathcal{T}_{\Gamma}$. We then define four core paths $P_{i}(x)(1 \leq i \leq 4)$ of $G(x)$ as $P_{1}(x)=P_{1}\left(z_{1}\right) \cup \bigcup_{i=1}^{j} e_{i}, P_{2}(x)=\bigcup_{i=1}^{p^{\prime}} P_{2}\left(z_{i}^{\prime}\right) \cup \bigcup_{i=1}^{q^{\prime}} e_{i}^{\prime}, P_{3}(x)=$ $P_{3}\left(z_{p}\right) \cup \bigcup_{i=k+1}^{q} e_{i}$, and $P_{4}(x)=\bigcup_{i=1}^{p} P_{2}\left(z_{i}\right) \cup \bigcup_{i=j+1}^{k} e_{i}$, as shown in Fig. 2(e). We define a straight line drawing $\Gamma$ of $G$ to be a canonical drawing of $G$ if the following $(a)$ and $(b)$ hold for $\Gamma$. (a) For each non-root $P$-node $x$ in $\mathcal{T}_{\Gamma}$, each core path $P_{i}(x)$ of $G(x)$ is drawn on a different line segment $L_{i}(x)$; and $(b)$ there is a primitive $P$-node $w$ in $\mathcal{T}_{\Gamma}$ such that the poles of $w$ appear on the outerface of $\Gamma$.

Let $L(\Gamma)$ denote the number of segments in the drawing $\Gamma$ of $G$. We call a line segment $l_{1}$ in $\Gamma$ to be collinear with another line segment $l_{2}$ in $\Gamma$ if $l_{1}$ and $l_{2}$ have the same slope, and the perpendicular distance between $l_{1}$ and $l_{2}$ is zero. For a node $x$ in $\mathcal{T}_{\Gamma}$, we use $\Gamma(x)$ to denote the drawing of $G(x)$ in $\Gamma$, and $\Gamma \backslash \Gamma(x)$ to denote the drawing obtained by deleting $\Gamma(x)$ from $\Gamma$. If $\Gamma$ is a canonical drawing of $G$, then we say that $\Gamma(x)$ is a canonical drawing of $G(x)$. We say that $\Gamma(x)$ shares a line segment 


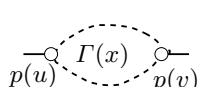

(a)

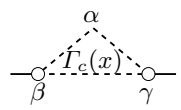

(b)

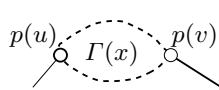

(c)

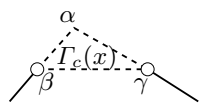

(d)

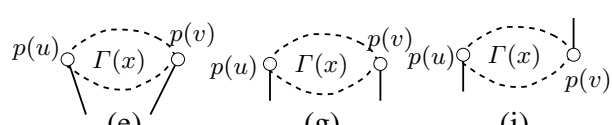

(e)

(g)

(i)

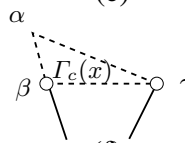

(f)

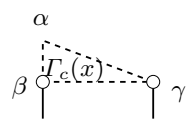

(h)

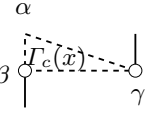

(j)

Fig. 3. All possible cases for computing $\Gamma_{c}(x)$ when $x$ is a primitive $P$-node

with $\Gamma \backslash \Gamma(x)$ if there is a line segment $l_{1}$ in $\Gamma(x)$ and a line segment $l_{2}$ in $\Gamma \backslash \Gamma(x)$ such that $l_{1}$ and $l_{2}$ are collinear and have a common end point.

\section{Biconnected Series-Parallel Graphs}

In this section we give our algorithm for computing a minimum segment drawing of a biconnected series-parallel graph $G$ with $\Delta(G)=3$. We first show that any drawing $\Gamma$ of $G$ can be transformed into a canonical drawing $\Gamma_{c}$ such that $L\left(\Gamma_{c}\right) \leq L(\Gamma)$. We then give a lower bound of $L(\Gamma)$, and describe our drawing algorithm.

We have the following lemma on transformation of a drawing into canonical drawing.

Lemma 1. Let $G$ be a biconnected series-parallel graph with $\Delta(G)=3$. Then for any straight-line drawing $\Gamma$ of $G$, a canonical drawing $\Gamma_{c}$ of $G$ can be computed such that $L\left(\Gamma_{c}\right) \leq L(\Gamma)$.

Proof. Let $x$ be a non-root $P$-node having poles $u$ and $v$ in $\mathcal{T}_{\Gamma}$. By Fact 1 , there is a sibling $Q$-node of $x$ preceding it and a sibling $Q$-node of $x$ following it in $\mathcal{T}_{\Gamma}$. Let $e(x)=\left(u^{\prime}, u\right)$ and $e^{\prime}(x)=\left(v^{\prime}, v\right)$ denote the two edges corresponding to these two $Q$ nodes respectively. Let $h(x)$ denote the height of $x$ in $\mathcal{T}_{\Gamma}$. Using induction on $h(x)$ we now prove that for each non-root $P$-node $x$ of $\mathcal{T}_{\Gamma}$, we can compute a canonical drawing $\Gamma_{c}(x)$ of $G(x)$ such that replacing $\Gamma(x)$ with $\Gamma_{c}(x)$ in $\Gamma$ does not increase $L(\Gamma)$.

For $h(x)=0$, we compute $\Gamma_{c}(x)$ by first drawing a triangle with three segments $L_{i}(x)(1 \leq i \leq 3)$, and then drawing the core path $P_{i}(x)$ on $L_{i}(x)(1 \leq i \leq 3)$. Considering all possible orientations of $l(e(x))$ and $l\left(e^{\prime}(x)\right)$, computation of $\Gamma_{c}(x)$ is shown in Fig. 3. In each case, we choose the line segment closed between $\alpha$ and $\beta$ as $L_{1}(x)$, the one closed between $\beta$ and $\gamma$ as $L_{2}(x)$, and the one closed between $\alpha$ and $\gamma$ as $L_{3}(x)$. We now show that, $L(\Gamma)$ will not increase if we replace $\Gamma(x)$ with $\Gamma_{c}(x)$. Since $G(x)$ is a simple cycle, any straight line drawing of $G(x)$ would require at least three line segments. Again, in any straight line drawing of $G, \Gamma(x)$ may share at most two line segments with $\Gamma \backslash \Gamma(x)$. Except for the case where $l(e(x))$ and $l\left(e^{\prime}(x)\right)$ are parallel (as in Fig. 3 $\mathrm{g}$ ) and (i)) or diverging (as in Fig. 3. e)), we have not reduced the number of line segments that might have been shared between $\Gamma(x)$ and $\Gamma \backslash \Gamma(x)$ as shown in Fig. 3(a)-(d). If $l(e(x))$ and $l\left(e^{\prime}(x)\right)$ are parallel or diverging as illustrated in Fig. 3(e)-(j), our drawing might have reduced this number by at most one if $\Gamma(x)$ had shared both the line-segments $l(e(x))$ and $l\left(e^{\prime}(x)\right)$. However, if $\Gamma(x)$ had shared both 


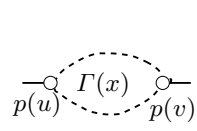

(a)

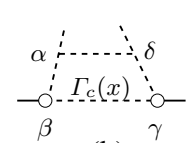

(b)

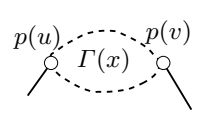

(c)

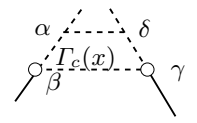

(d)

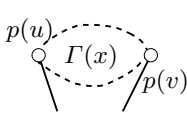

(e)

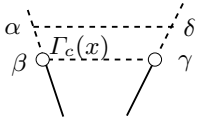

(f)

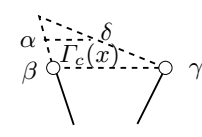

(g)

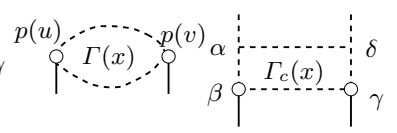

(h)

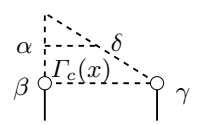

(j)

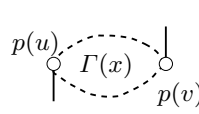

(k)

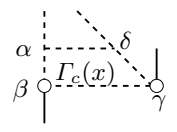

(1)

Fig. 4. All possible cases for computing $\Gamma_{c}(x)$ when $h(x)>0$

the line segments $l(e(x))$ and $l\left(e^{\prime}(x)\right)$, then in every case, any straight line drawing of $G(x)$ would require at least four segments, and we have reduced this number by at least one. Hence, replacing $\Gamma(x)$ with $\Gamma_{c}(x)$ would not increase $L(\Gamma)$ in any of the cases.

We now assume that $h(x)>0$ and $\Gamma_{c}(w)$ has been computed for all the descendant $P$-nodes $w$ of $x$. To compute $\Gamma_{c}(x)$, we first draw a quadrangle with four line segments $L_{i}(x)(1 \leq i \leq 4)$, in such a way that $L_{2}(x)$ is the line segment closed between $p(u)$ and $p(v)$. Based on different orientation of $l(e(x))$ and $l\left(e^{\prime}(x)\right)$, the quadrangle is illustrated in Fig. 4. In every case, we choose the line segment closed between $\alpha$ and $\beta$ as $L_{1}(x)$, the one closed between $\beta$ and $\gamma$ as $L_{2}(x)$, the one between $\gamma$ and $\delta$ as $L_{3}$ and the one between $\alpha$ and $\delta$ as $L_{4}(x)$. We then draw the core path $P_{i}(x)$ along $L_{i}(x)(1 \leq i \leq 4)$. Finally, for each child $P$-node $w$ of $y$, we add $\Gamma_{c}(w)$ by making $L_{2}(w)$ and $L_{4}(x)$ collinear. Similarly, for each child $P$-node $w$ of $y^{\prime}$, we draw $\Gamma_{c}(w)$ by making $L_{2}(w)$ and $L_{2}(x)$ collinear. The fact that replacing $\Gamma(x)$ with $\Gamma_{c}(x)$ does not increase $L(\Gamma)$ can be understood as follows. Let $\Gamma^{\prime}(x)$ denote the drawing obtained by considering $\Gamma_{c}(x)$ and the two line segments $l(e(x))$ and $l\left(e^{\prime}(x)\right)$. Let $G^{\prime}(x)$ denote the underlying graph of $\Gamma^{\prime}(x)$. If $l(e(x))$ and $l\left(e^{\prime}(x)\right)$ are collinear or converging as illustrated in Fig. 4(a)-(d), then for each degree two vertex $v^{\prime}$ of $G^{\prime}(x)$, the two incident edges of $v^{\prime}$ are collinear in $\Gamma^{\prime}(x)$ with the exception that for each primitive $P$-node, the incident edges of exactly one degree two vertex are non-collinear. Again for each degree three vertex $v^{\prime}$ of $G^{\prime}(x)$, exactly two of the three incident edges are collinear in $\Gamma^{\prime}(x)$. Thus, $\Gamma^{\prime}(x)$ has the maximum possible sharing between the drawings of the edges of $G^{\prime}(x)$, and replacing $\Gamma(x)$ with $\Gamma_{c}(x)$ will not increase $L(\Gamma)$. Similarly, if $l(e(x))$ and $l\left(e^{\prime}(x)\right)$ are parallel with the angle between them being $0^{\circ}$ as shown in Fig. 4 (h)-(j), and if $x$ has a child $S$-node with at least two child $P$-nodes, then $\Gamma^{\prime}(x)$ will have the maximum possible sharing between the drawings of the edges of $G(x)$, and replacing $\Gamma(x)$ with $\Gamma_{c}(x)$ will not increase $L(\Gamma)$. On the other hand, if each child $S$-node of $x$ has at most one child $P$-node, then the three incident edges of $v$ are pairwise noncollinear in $\Gamma^{\prime}(x)$ as shown in Fig. 4(j) and Fig. 5(c). However, $L(\Gamma)$ will not increase even in this case. If both the line segments $l(e(x))$ and $l\left(e^{\prime}(x)\right)$ were shared by some line-segment in $\Gamma(x)$, then either of the following $(a)$ and $(b)$ will hold. $(a)$ There is at least one descendant non-primitive $P$-node $x^{\prime}$ of $x$ such that at either of the two poles of $x^{\prime}$, all the three incident edges are pairwise non-collinear as shown in Fig. 5(a); and (b) there is at least one descendant primitive $P$-node $x^{\prime \prime}$ of $x$ such that $\Gamma\left(x^{\prime \prime}\right)$ uses four line-segments as shown in Fig. 5 b). In both the cases, replacing $\Gamma(x)$ with $\Gamma_{c}(x)$ 


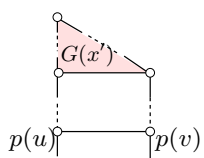

(a)

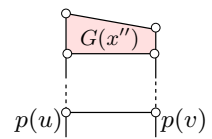

(b)

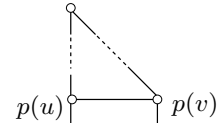

(c)

Fig. 5. (a) and (b) Two drawings of $G(x)$ that shares both the line segments $l(e(x))$ and $l\left(e^{\prime}(x)\right)$ with the rest of the drawing, (c) a canonical drawing $\Gamma_{c}(x)$ of $G(x)$

does not increase $L(\Gamma)$. The reasoning for the cases where line segments $l(e(x))$ and $l\left(e^{\prime}(x)\right)$ are parallel with the angle between them being $180^{\circ}$ (as shown in Fig. 4 (k) and (1)), or where $l(e(x))$ and $l\left(e^{\prime}(x)\right)$ are diverging (as shown in Fig.4(e)-(g)) follows from similar arguments.

It now remains for us to show that we can obtain a primitive $P$-node $w$ in $\mathcal{T}_{\Gamma}$ such that the poles of $w$ appear on the outerface of $\Gamma_{c}$. One can observe that for each nonroot $P$-node $x$ of $\mathcal{T}_{\Gamma}$, there is a primitive $P$-node $w$ in the subtree of $\mathcal{T}_{\Gamma}$ rooted at $x$ such that the poles of $w$ appear on the outerface of $\Gamma_{c}(x)$. Let $r$ denote the root of $\mathcal{T}$. Let $y_{1}, y_{2}$ and $y_{3}$ denote the three children of $r$ in $\mathcal{T}$. We first consider the nodes $y_{1}$ and $y_{2}$ as the two children of a temporary $P$-node $x^{\prime}$ and compute $\Gamma_{c}\left(x^{\prime}\right)$ in the same way as described in the inductive step above. We then replace $\Gamma\left(x^{\prime}\right)$ with $\Gamma_{c}\left(x^{\prime}\right)$, and this does not increase $L(\Gamma)$. We then take a single line segment and draw on it all the edges corresponding to the child $Q$-nodes of $y_{3}$ along with all the paths $P_{2}(z)$ for each child $P$-node $z$ of $y_{3}$. Let $\Gamma^{\prime}\left(y_{3}\right)$ denote this drawing of $G\left(y_{3}\right)$. We then compute $\Gamma_{c}$ by merging $\Gamma_{c}\left(x^{\prime}\right)$ with $\Gamma^{\prime}\left(y_{3}\right)$. The details of the proof that the merging of $\Gamma_{c}\left(x^{\prime}\right)$ and $\Gamma^{\prime}\left(y_{3}\right)$ does not increase $L(\Gamma)$ is omitted in this extended abstract since the arguments are similar to those given in the induction step above. One can also observe that after performing the merging of $\Gamma_{c}\left(x^{\prime}\right)$ and $\Gamma^{\prime}\left(y_{3}\right)$, we will obtain the poles of a primitive $P$-node in the outerface of $\Gamma_{c}$.

Lemma 1 implies that any straight line drawing $\Gamma$ of $G$ requires at least $L\left(\Gamma_{c}\right)$ line segments where $\Gamma_{c}$ is a canonical drawing obtained by transforming $\Gamma$. We therefore give here a lower bound of $L\left(\Gamma_{c}\right)$. For clarity of notations, we use $\mathcal{T}$ instead of $\mathcal{T}_{\Gamma_{c}}$ to denote an $S P Q$-tree corresponding to $\Gamma_{c}$. Since there is always a primitive $P$-node $w$ in $\mathcal{T}$ such that the poles of $w$ appear on the outerface of $\Gamma_{c}$, we assume that the root of $\mathcal{T}$ has two child $S$-nodes that are primitive in $\mathcal{T}$. We first have the following lemma.

Lemma 2. Let $G$ be a biconnected series-parallel graph with $\Delta(G)=3$. Let $\Gamma_{c}$ be a canonical drawing of $G$. Let $\mathcal{T}$ be an $S P Q$-tree of $G$ corresponding to $\Gamma_{c}$. Then for a non-root $P$-node $x$ in $\mathcal{T}, L\left(\Gamma_{c}(x)\right) \geq P_{x}+N_{x}+1$.

Proof. We use induction on $P_{x}$. In the basis case, $P_{x}=1$, i.e., $x$ is a primitive $P$-node. Hence $N_{x}=1$ and $P_{x}+N_{x}+1=3$. Since $G(x)$ is a simple cycle when $x$ is primitive and any straight line drawing of a cycle requires at least three segments, the claim holds.

We now assume that $P_{x}>0$ and the claim holds for every $P$-node $w$ in $\mathcal{T}$ having $P_{w}<P_{x}$. Hence $L\left(\Gamma_{c}(w)\right) \geq P_{w}+N_{w}+1$. We now take a child $P$-node $w$ of $x$ and delete the drawing $\Gamma_{c}(w)$ from $\Gamma_{c}$. Let $G^{\prime}$ denote the underlying graph of this drawing $\Gamma_{c} \backslash \Gamma_{c}(w)$. The graph $G^{\prime}$ is not necessarily a biconnected series-parallel graph. Let $u$ and $v$ be the two poles of $w$ in $\mathcal{T}$. In order to ensure that we are working with a 


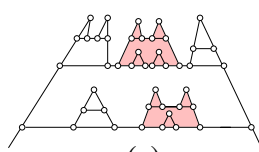

(a)

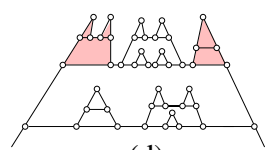

(d)

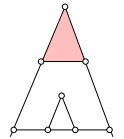

(g)

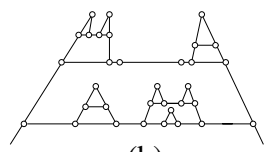

(b)

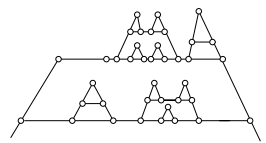

(e)

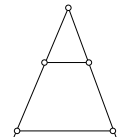

(i)

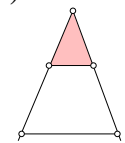

(j)

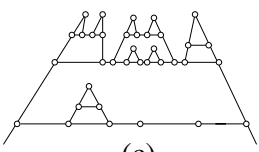

(c)

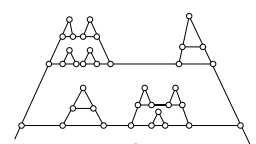

(f)

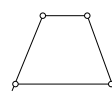

(k)

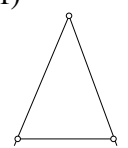

(1)

Fig. 6. Cases in the induction step of the proof of Lemma2 $2 \Gamma_{c}(w)$ shown highlighted in each case.

biconnected series-parallel graph, we now add an edge $(u, v)$ to $G^{\prime}$, and a new line segment between the points $p(u)$ and $p(v)$ in $\Gamma_{c}$. We then replace the node $w$ in $\mathcal{T}$ with a $Q$-node representing the edge $(u, v)$, and rename the node $x$ as $x^{\prime}$. Let $\Gamma^{\prime}$ denote this newly computed drawing of $G\left(x^{\prime}\right)$. Since $P_{x^{\prime}}<P_{x}$, we have $L\left(\Gamma_{c}\left(x^{\prime}\right) \geq P_{x^{\prime}}+N_{x^{\prime}}+1\right.$. We now have the following two cases to consider.

Case 1. $\Gamma^{\prime}$ is canonical.

This case may occur in either of the following two subcases. (i) $p>2$ and $w=z_{i}$ $(2 \leq i \leq p-1)$, as illustrated in Fig. 6) (a) and (b); and (ii) $p^{\prime} \geq 1$ and $w=z_{i}^{\prime}$ $\left(1 \leq i \leq p^{\prime}\right)$, as illustrated in Fig. 6(a) and (c). In both the subcases, $\Gamma_{c}(w)$ had exactly one line segment shared with $\Gamma_{c}(x)$. Thus, $L\left(\Gamma^{\prime}\right)=L\left(\Gamma_{c}(x)\right)-L\left(\Gamma_{c}(w)\right)+1$. Again, since $\Gamma^{\prime}$ is canonical, $\left.L\left(\Gamma^{\prime}\right)\right)=L\left(\Gamma_{c}\left(x^{\prime}\right)\right)$. By induction hypothesis we have $L\left(\Gamma_{c}(x)\right) \geq P_{x^{\prime}}+N_{x^{\prime}}+1+P_{w}+N_{w}+1-1=P_{x}+N_{x}+1$.

Case 2. $\Gamma^{\prime}$ is not canonical.

Here we have the following three subcases.

(i) $p>2$ and either $w=z_{1}$ or $w=z_{p}$, as illustrated in Fig.6(d) and (e), (ii) $p=p^{\prime}=1$ and $w=z_{1}$, as illustrated in Fig. 6. (g) and (h); and (iii) $p=1, p^{\prime}=0$ and $w=z_{1}$, as illustrated in Fig. 6(j) and (k). We omit the proofs for the second and third subcase in this extended abstract. For the subcase $2(i), \Gamma_{c}(w)$ had exactly two line segments shared with $\Gamma_{c}(x)$. Thus, $L\left(\Gamma^{\prime}\right)=L\left(\Gamma_{c}(x)\right)-L\left(\Gamma_{c}(w)\right)+2$. Since $\Gamma^{\prime}$ is not canonical, we now make it canonical by making $L_{1}\left(z_{2}\right)$ collinear with $L_{1}\left(x^{\prime}\right)$ if $w=z_{1}$ or, by making $L_{3}\left(z_{p-1}\right)$ collinear with $L_{3}\left(x^{\prime}\right)$ if $w=z_{p}$ as illustrated in Fig. 6(f). One can observe that, in both the cases, the number of line segments decreases by exactly one in $\Gamma_{c}\left(x^{\prime}\right)$. Thus, $L\left(\Gamma_{c}\left(x^{\prime}\right)\right)=L\left(\Gamma^{\prime}\right)-1$. By induction hypothesis we then have $L\left(\Gamma_{c}(x)\right) \geq$ $P_{x^{\prime}}+N_{x^{\prime}}+1+P_{w}+N_{w}+1-1=P_{x}+N_{x}+1$.

We now have the following theorem.

Theorem 1. Let $G$ be a biconnected series-parallel graph with $\Delta(G)=3$. Let $\Gamma_{c}$ be a canonical drawing of $G$. Let $\mathcal{T}$ be an $S P Q$-tree of $G$ corresponding to $\Gamma_{c}$. Let $P_{\mathcal{T}}$ and $N_{\mathcal{T}}$ denote the number of $P$-nodes and the number of primitive $P$-nodes respectively in $\mathcal{T}$. Then the following (a) and (b) hold. (a) $L\left(\Gamma_{c}\right) \geq P_{\mathcal{T}}+N_{\mathcal{T}}+2$, if every $S$-node in $\mathcal{T}$ has at most one child $P$-node; and (b) $L\left(\Gamma_{c}\right) \geq P_{\mathcal{T}}+N_{\mathcal{T}}+1$, otherwise. 


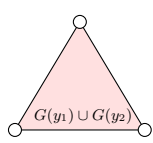

(a)

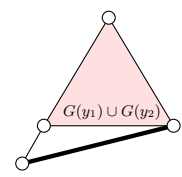

(b)

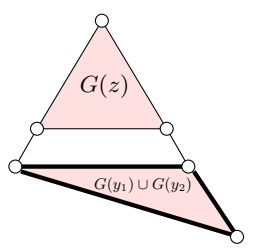

(c)

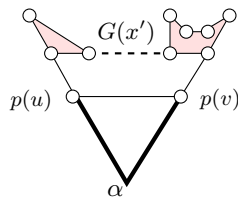

(d)

Fig. 7. (a) The drawing of $G\left(y_{1}\right) \cup G\left(y_{2}\right)$, (b) $\Gamma_{c}$ if $y_{3}$ is primitive, (c) $\Gamma_{c}$ if $y_{3}$ has exactly one child $P$-node, and (d) $\Gamma_{c}$ if $y_{3}$ has at least two child $P$-nodes

Proof. Let $r$ be the root of $\mathcal{T}$. Let $y_{1}, y_{2}$ and $y_{3}$ be the three children of $r$ in $\mathcal{T}$. Since $\Gamma_{c}$ is a canonical drawing, we assume that $y_{1}$ and $y_{2}$ are primitive in $\mathcal{T}$. Since $G$ is a simple graph, exactly one of $y_{1}, y_{2}$ and $y_{3}$ can be a $Q$-node in $\mathcal{T}$. Thus, if there is a $Q$-node among $y_{1}, y_{2}$ and $y_{3}$, then we assume that $y_{2}$ is the $Q$-node. The proofs of the claims $(a)$ and $(b)$ are given below.

(a) We have the following two cases to consider here.

Case 1. $y_{3}$ is primitive in $\mathcal{T}$.

Since $G\left(y_{1}\right) \cup G\left(y_{2}\right)$ is a cycle, at least three line segments are required to draw $G\left(y_{1}\right) \cup G\left(y_{2}\right)$ in $\Gamma_{c}$ as illustrated in Fig.7(a). Since $G\left(y_{3}\right)$ is a path, at least one new line segment is required to draw $G\left(y_{3}\right)$ along with $G\left(y_{1}\right) \cup G\left(y_{2}\right)$ in $\Gamma_{c}$ as illustrated through the thick line segment in Fig.7(b). Since $P_{\Gamma}=1, N_{\Gamma}=1$, and $P_{\Gamma}+N_{\Gamma}+2=4$, we thus have $L\left(\Gamma_{c}\right)=4 \geq P_{\Gamma}+N_{\Gamma}+2$.

Case 2. $y_{3}$ is not primitive in $\mathcal{T}$.

Let $z$ denote the child $P$-node of $y_{3}$ in $\mathcal{T}$. By Lemma2 $L\left(\Gamma_{c}(z)\right) \geq P_{z}+N_{z}+1$. One can observe that $G\left(y_{1}\right) \cup G\left(y_{2}\right)$ is connected with $G\left(y_{3}\right)$ through the two edges incident to the two poles of $G\left(y_{3}\right)$. Hence, any drawing of $G\left(y_{1}\right) \cup G\left(y_{2}\right)$ can share at most two segments with the drawing $\Gamma_{c}(z)$. However, as shown in the proof of Lemma 1 since each $S$-node in $\mathcal{T}$ has at most one child $P$-node, we cannot draw the line segments $L_{1}(z)$ and $L_{3}(z)$ as converging in the exterior of $\Gamma_{c}(z)$ without increasing $L\left(\Gamma_{c}(z)\right)$. Since $L_{1}(z)$ and $L_{3}(z)$ are converging in the interior of $\Gamma_{c}(z)$, at least two new segments are required to draw $G\left(y_{1}\right) \cup G\left(y_{2}\right)$ along with $G\left(y_{3}\right)$ as shown through the thick line segments in Fig. 7(c). Thus, $L\left(\Gamma_{c}\right) \geq P_{z}+N_{z}+1+2$. Since $P_{\Gamma}=P_{z}+1, N_{\Gamma}=N_{z}$, we thus have $L\left(\Gamma_{c}\right) \geq P_{\Gamma}+N_{\Gamma}+1$.

(b) In this case $y_{3}$ has at least two child $P$-nodes in $\mathcal{T}$. We consider $y_{2}$ and $y_{3}$ as the two $S$-nodes of a temporary $P$-node $x^{\prime}$, and compute $\Gamma_{c}\left(x^{\prime}\right)$ as described in the proof of Lemma11. By Lemma2, $L\left(\Gamma_{c}\left(x^{\prime}\right)\right) \geq P_{x^{\prime}}+N_{x^{\prime}}+1$. As shown in the proof of Lemma1 since at least one $S$-node in $\mathcal{T}$ has two child $P$-nodes, we can draw the line segments $L_{1}\left(x^{\prime}\right)$ and $L_{3}\left(x^{\prime}\right)$ as converging in the exterior of $\Gamma_{c}\left(x^{\prime}\right)$ as illustrated in Fig. 7 d $)$. Let $\alpha$ denote the point where $L_{1}\left(x^{\prime}\right)$ and $L_{3}\left(x^{\prime}\right)$ converges. Let $u$ and $v$ denote the poles of $r$. Since $y_{3}$ is not a $Q$-node, we can now complete the drawing of $G\left(y_{3}\right)$ on the two line segments closed between $p(u), \alpha$ and $\alpha, p(v)$ without requiring any new line segment. Since $P_{x^{\prime}}=P_{\Gamma}, N_{x^{\prime}}=N_{\Gamma}$, we have $L\left(\Gamma_{c}\right) \geq P_{x^{\prime}}+N_{x^{\prime}}+1=P_{\Gamma}+N_{\Gamma}+1$.

We now present our main result on minimum segment drawing in the following theorem. 


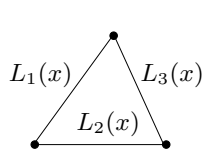

(a)

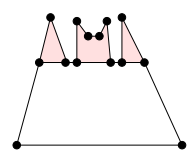

(e)

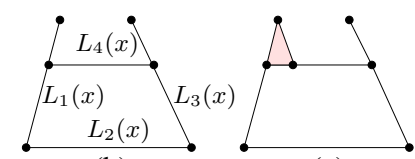

(b)

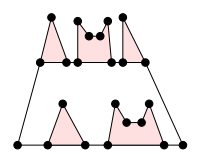

(f)

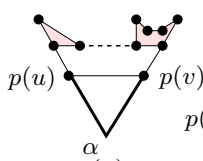

(g)

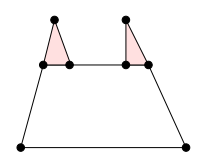

(d)

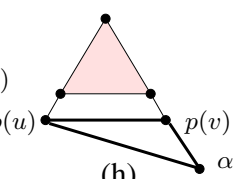

(h)

Fig. 8. (a) Drawing of $G(x)$ for a primitive $P$-node $x$, (b) the quadrangle for a non-root and non-primitive $P$-node $x$, (c)-(f) completing the drawing $\Gamma(x)$ for a non-root and non-primitive $P$-node $x,(\mathrm{~g}) \Gamma$ when there is a suitable root in $\mathcal{T}$, and (h) $\Gamma$ when there is no suitable root in $\mathcal{T}$

Theorem 2. Let $G$ be a biconnected series-parallel graph with $\Delta(G)=3$. Then a minimum segment drawing of $G$ can be computed in linear time.

Proof. We first compute a planar straight line drawing $\Gamma$ of $G$ in linear time and then show that $\Gamma$ is a minimum segment drawing of $G$.

Let $\mathcal{T}$ be an $S P Q$-tree of $G$ rooted at an arbitrary $P$-node $r$. Let $y_{1}, y_{2}$ and $y_{3}$ denote the three children of $r$ in $\mathcal{T}$. Since $G$ is a simple graph, at most one of $y_{1}, y_{2}$ and $y_{3}$ can be a $Q$-node. Thus, if there is a $Q$-node among $y_{1}, y_{2}$ and $y_{3}$, then we assume that $y_{2}$ is the $Q$-node. In order to compute a minimum segment drawing of $G$, we want the following two conditions to hold for $y_{1}, y_{2}$ and $y_{3}$ in $\mathcal{T}$. (a) $y_{1}$ and $y_{2}$ are primitive in $\mathcal{T}$; and (b) $y_{3}$ has at least two child $P$-nodes in $\mathcal{T}$. If these conditions hold for the three children of our arbitrarily chosen root $r$, then we are done. Otherwise, we search for such a $P$-node $r$ in $\mathcal{T}$. If there is such a $P$-node $r$ in $\mathcal{T}$, then there is an $S$-node $x$ in $\mathcal{T}$ such that $x$ has at least two child $P$-nodes, one of which is primitive. We can search for such an $S$-node $x$ in $\mathcal{T}$ in linear time. If we find such an $S$-node $x$ in $\mathcal{T}$, then the child primitive $P$-node $r$ of $x$ will be our desired root of $\mathcal{T}$. If we fail to find any such $S$-node $x$ in $\mathcal{T}$, then each $S$-node in $\mathcal{T}$ has at most one child $P$-node in $\mathcal{T}$. We then choose any primitive $P$-node $r$ in $\mathcal{T}$ as the root of $\mathcal{T}$. We now compute $\Gamma$ in a bottom up traversal of $\mathcal{T}$. At first, in each non-root $P$-node $x$ of $\mathcal{T}$, we compute a canonical drawing $\Gamma(x)$ of $G(x)$ from the previously computed canonical drawings $\Gamma(w)$ of $G(w)$ for each child $P$-node $w$ of $x$. Then we compute $\Gamma=\Gamma(r)$ for the root $r$ of $\mathcal{T}$. We describe this construction inductively. For a primitive $P$-node $x$ in $\mathcal{T}$, we draw the three core paths $P_{i}(x)(1 \leq i \leq 3)$ of $G(x)$ on three line segments $L_{i}(x)$ $(1 \leq i \leq 3)$ such that each line segment $L_{i}(x)$ is closed between the end vertices of $P_{i}(x)(1 \leq i \leq 3)$, as illustrated in Fig. 8(a). For a non-root and non-primitive $P$-node $x$ in $\mathcal{T}$, we first draw the four core paths $P_{i}(x)(1 \leq i \leq 4)$ on four line segments $L_{i}(x)(1 \leq i \leq 4)$ such that each line segment $L_{i}(x)$ is closed between the end vertices of $P_{i}(x)(1 \leq i \leq 4)$, as illustrated in Fig. 8 b). For each child $P$-node $w$ of $x$, we now add $\Gamma(w)$ to this quadrangle and complete the drawing $\Gamma(x)$ as follows. At first, we draw $\Gamma\left(z_{1}\right)$ by making $L_{1}\left(z_{1}\right)$ collinear with $L_{1}(x)$, and $L_{2}\left(z_{1}\right)$ collinear with $L_{4}(x)$ as shown in Fig. 8 (c). Next we draw $\Gamma\left(z_{p}\right)$ by making $L_{3}\left(z_{p}\right)$ collinear with $L_{3}(x)$ and $L_{2}\left(z_{p}\right)$ collinear with $L_{4}(x)$ as shown in Fig. $8(d)$. Finally, for each $w=z_{i}$ 
$(2 \leq i \leq p-1)$, we draw $\Gamma(w)$ by making $L_{2}(w)$ collinear with $L_{4}(x)$ as shown in Fig. 8 (e), and for each $w=z_{i}^{\prime}\left(1 \leq i \leq p^{\prime}\right)$, we draw $\Gamma(w)$ by making $L_{2}(w)$ collinear with $L_{2}(x)$ as shown in Fig. 8 (f). We finally assume that $x$ is the root $P$-node of $\mathcal{T}$. Let $u$ and $v$ denote the poles of $x$ in $\mathcal{T}$. To compute $\Gamma=\Gamma(x)$, we first consider $y_{2}$ and $y_{3}$ as the children of a temporary $P$-node $x^{\prime}$ and compute the canonical drawing $\Gamma\left(x^{\prime}\right)$ in the same way as described in the inductive case above. We now have the following two cases to consider. We first consider the case where $\mathcal{T}$ has a suitable root as described earlier. By construction, we will have two line segments in $\Gamma\left(x^{\prime}\right)$ in this case, namely $L_{1}\left(x^{\prime}\right)$ and $L_{3}\left(x^{\prime}\right)$ that can be drawn as converging in the exterior of $\Gamma\left(x^{\prime}\right)$. Let $\alpha$ denote the point where $L_{1}\left(x^{\prime}\right)$ and $L_{3}\left(x^{\prime}\right)$ converge. We then draw the graph $G\left(y_{3}\right)$ on the two line segments closed between $p(u), \alpha$ and $\alpha, p(v)$, as shown in Fig. 8 g). We next consider the case where $\mathcal{T}$ does not have a suitable root $r$ as described earlier. In this case, we take a point $\alpha$ in the exterior of $\Gamma\left(x^{\prime}\right)$ such that the points $p(u), p(v)$ and $\alpha$ form a triangle as shown in Fig. 8 (h). We then draw the graph $G\left(y_{3}\right)$ on the two line segments closed between $p(u), \alpha$ and $\alpha, p(v)$. Clearly, the drawing $\Gamma$ described above can be computed in linear time. We omit the details of this proof of time complexity in this extended abstract.

We now prove that $\Gamma$ has the minimum number of segments. We first prove that for each non-root $P$-node $x$ of $\mathcal{T}$, we draw $G(x)$ on $P_{x}+N_{x}+1$ segments. We give here an inductive proof by taking induction on the height $h(x)$ of $x$. For $h(x)=0, P_{x}+N_{x}+$ $1=3$. We have drawn $G(x)$ on three line segments, and our claim holds for $h(x)=0$. We now consider $h(x)>0$ and $x$ is a non-root and non-primitive $P$-node. While computing $\Gamma(x)$, we have drawn $G\left(y^{\prime}\right)$ in such a way that all the edges corresponding to the child $Q$-nodes of $y^{\prime}$ were drawn on a single segment, and $L_{2}\left(z_{i}^{\prime}\right)$ for each $G\left(z_{i}^{\prime}\right)$ was drawn on the same segment. Thus the number of segments in this drawing of $G\left(y^{\prime}\right)$ is $P_{y}^{\prime}+N_{y}^{\prime}+p^{\prime}-\left(p^{\prime}-1\right)=P_{y}^{\prime}+N_{y}^{\prime}+1$. Similarly, $G(y)$ was first drawn on $P_{y}+N_{y}+1$ segments and then the path $\bigcup_{i=1}^{j} e_{i}$ was drawn on the same segment as $L_{1}\left(z_{1}\right)$ and the path $\bigcup_{i=k+1}^{q} e_{i}$ was drawn on the same segment as $L_{3}\left(z_{p}\right)$. Here $e_{j}$ and $e_{k}$ are the two edges corresponding to the two $Q$-nodes immediately preceding $z_{1}$ and $z_{p}$ respectively in $\mathcal{T}$. Since we had reused an already drawn segment, this last operation did not increase the number of segments. We finally had merged these drawings of $G(y)$ and $G\left(y^{\prime}\right)$ together to get a drawing of $G(x)$ on $P_{y}+N_{y}+1+P_{y}^{\prime}+N_{y}^{\prime}+1=$ $\left(P_{y}+P_{y}^{\prime}+1\right)+N_{x}+1=P_{x}+N_{x}+1$ segments. Finally, in the root node, we did not draw any new line segment if a suitable root $r$ was found for $\mathcal{T}$, otherwise we had drawn exactly one new line segment. Thus we had drawn $\Gamma$ on $P_{x}^{\prime}+N_{x}^{\prime}+1$ segments in the first case, and on $P_{x}^{\prime}+N_{x}^{\prime}+2$ segments in the second case. Since $P_{\Gamma}=P_{x^{\prime}}, N_{\Gamma}=N_{x^{\prime}}$, we have ultimately drawn $\Gamma$ on $P_{\Gamma}+N_{\Gamma}+2$ segments if each $S$-node in $\mathcal{T}$ had at most one child $P$-node, and on $P_{\Gamma}+N_{\Gamma}+1$ segments otherwise. Both these quantities matches the bound given on $L(\Gamma)$ in Theorem 1 and this completes the proof.

\section{Series-Parallel Graphs with Cut Vertices}

So far we have dealt with biconnected series-parallel graphs with the maximum degree three. However, the same idea can be adopted to compute a minimum segment drawing of a series-parallel graph $G$ that contains cut vertices. In this case, we first compute 
the blocks of $G$. Each block of $G$ is either a single edge or a series-parallel graph $G^{\prime}$ which can be decomposed similarly to the pertinent subgraph $G(x)$ of a $P$-node $x$ in the $S P Q$-tree of a biconnected series-parallel graph with the maximum degree three. For each such graph $G(x)$, we then compute a canonical drawing of $G(x)$. Next we add a single line segment aligned with the path $P_{2}(x)$ of each block $G(x)$ and complete the drawing of $G$.

\section{Conclusion}

In this paper we have given a linear-time algorithm for computing minimum segment drawings of series-parallel graphs with the maximum degree three. To the best of our knowledge, this is the first result in this problem focusing on an important subclass of planar graphs. It remains as our future work to achieve similar results for wider subclasses of planar graphs.

\section{Acknowledgements}

This work is based on the M.Sc. Engineering Thesis [7] completed in the Department of Computer Science and Engineering, Bangladesh University of Engineering and Technology (BUET). We acknowledge BUET for supporting this work throughout.

\section{References}

1. Dujmović, V., Eppstein, D., Suderman, M., Wood, D.R.: Drawings of planar graphs with few slopes and segments. Comput. Geom. 38(3), 194-212 (2007)

2. Fáry, I.: On straight line representation of planar graphs. Acta Sci. Math. Szeged 11, 229-233 (1948)

3. de Fraysseix, H., Pach, J., Pollack, R.: How to draw a planar graph on a grid. Combinatorica 10(1), 41-51 (1990)

4. Nishizeki, T., Rahman, M.S.: Planar Graph Drawing. Lecture Notes Series on Computing, vol. 12. World Scientific Publishing Co., Singapore (2004)

5. Purchase, H.C.: Which aesthetic has the greatest effect on human understanding? In: DiBattista, G. (ed.) GD 1997. LNCS, vol. 1353, pp. 248-261. Springer, Heidelberg (1997)

6. Rahman, M.S., Egi, N., Nishizeki, T.: No-bend orthogonal drawings of series-parallel graphs. In: Healy, P., Nikolov, N.S. (eds.) GD 2005. LNCS, vol. 3843, pp. 409-420. Springer, Heidelberg (2006)

7. Samee, M.A.H.: Minimum Segment Drawings of Series-Parallel Graphs with the Maximum Degree Three. M.Sc. Engineering Thesis, Department of Computer Science and Engineering, Bangladesh University of Engineering and Technology (July 2008)

8. Schnyder, W.: Embedding planar graphs on the grid. In: First ACM-SIAM Symp. on Discrete Algorithms, pp. 138-148 (1990)

9. Stein, K.S.: Convex maps. Amer. Math. Soc. 2, 464-466 (1951)

10. Wagner, K.: Bemerkungen zum vierfarbenproblem. Jahresber. Deutsch. Math-Verein. 46, 26-32 (1936) 